\title{
ON THE HEAT KERNEL COMPARISON THEOREMS FOR MINIMAL SUBMANIFOLDS
}

\author{
STEEN MARKVORSEN
}

\begin{abstract}
In [3], Cheng, Li and Yau proved comparison theorems (upper bounds) for the heat kernels on minimal submanifolds of space forms. In the present note we show that these comparison theorems : ('gether with a series of corollaries remain true for minimal submanifolds in ambient spaces with just an upper bound on the sectional curvature.
\end{abstract}

1. Introduction. Let $M^{m}$ be a minimally immersed submanifold of $N^{n}$. For a given point $p \in M$ we define the normal range $U(p)$ to be the complement of the cut locus of $p$ in $N$. Let $r_{p}(\cdot)$ denote the distance function from $p$ in $N$. The ball $B_{R}(p)=\left\{x \in N \mid r_{p}(x) \leqslant R\right\}$ is then regular if $B_{R}(p) \subset U(p)$ and (when $\sup K_{N}$ $=b>0) R \leqslant \pi / 2 \sqrt{b}$.

Let $D \subset M^{m}$ be a compact domain of $M$ containing $p$. Following [3] we denote the $p$-centered heat kernels on $D$ by $H(p, y, t)$ (with Dirichlet boundary condition) and $K(p, y, t)$ (with Neumann boundary condition) respectively. If $\tilde{D}_{R}^{b}(\tilde{p})$ denotes the totally geodesic disc with center $\tilde{p}$, radius $R$ and dimension $m$ in a space form $\tilde{N}_{b}^{n}$ of constant curvature $b \in \mathbf{R}$, then the $\tilde{p}$-centered heat kernels on $\tilde{D}$ only depend on $r_{\tilde{p}}(\cdot)$ and $t$; hence we may, and do, write them as $\tilde{H}_{R}^{b}\left(r_{\tilde{p}}(y), t\right)$ and $\tilde{K}_{R}^{b}\left(r_{\tilde{p}}(y), t\right)$ respectively.

We can now formulate the extended comparison theorems for $H$ and $K$ as follows.

THEOREM 1. Let $M^{m}$ be a minimally immersed submanifold of $N^{n}$ with $K_{N} \leqslant b$. Let $D$ be a compact domain in $M$.

(i) If $D$ is contained in a regular ball $B_{R}(p) \subset N$ for some $p \in D$, then the Dirichlet heat kernel on $D$ satisfies

$$
H(p, y, t) \leqslant \tilde{H}_{R}^{b}\left(r_{p}(y), t\right) \quad \text { for all } y \in D \text { and } t \in \mathbf{R}_{+} .
$$

(ii) If $D=B_{R}(p) \cap M$ for some (not necessarily regular) ball $B_{R}(p) \subset U(p) \subset N$, $p \in D$, and (if $b>0) R \leqslant \pi / \sqrt{b}$, then the Neumann heat kernel on $D$ satisfies

$$
K(p, y, t) \leqslant \tilde{K}_{R}^{b}\left(r_{p}(y), t\right) \quad \text { for all } y \in D \text { and } t \in \mathbf{R}_{+} .
$$

This type of theorem was first proved by Cheng, Li and Yau for space form ambient spaces $N^{n}$ (cf. [3, Theorems 1 to 5]). The extension to ambient spaces with

Received by the editors July 12, 1985.

1980 Mathematics Subject Classification. Primary 53C42; Secondary 53C20, 58G11.

(C)1986 American Mathematical Society $0002-9939 / 86 \$ 1.00+\$ .25$ per page 
variable curvature is essentially a consequence of the following result, which may be proved by standard index comparison theory (cf. [4, Proposition 8]).

Proposition 2. We make the same assumptions as in Theorem 1. Let $F:[0, R] \rightarrow \mathbf{R}$ be a smooth function with $F^{\prime}(r) \geqslant 0$ for all $r \in[0, R]$, and let $\left\{X_{j}\right\}, 1 \leqslant j \leqslant m$, be an orthonormal basis of $T_{q} D$. Then the Laplacian $\Delta_{D}$ on $D$ satisfies the following inequality at $q$ :

$$
\begin{aligned}
\Delta_{D}\left(\left.F \circ r_{p}\right|_{D}\right) \geqslant & \left(F^{\prime \prime}(r)-F^{\prime}(r) h_{b}(r)\right) \sum_{j=1}^{m}\left\langle\operatorname{grad} r, X_{j}\right\rangle^{2} \\
& +m F^{\prime}(r) h_{b}(r),
\end{aligned}
$$

where $h_{b}(r)$ is the constant mean curvature of any distance sphere of radius $r$ in a space form of constant curvature $b$.

For the proof of Theorem 1 we also need the following special version of a result due to Cheeger and Yau [1, Lemma 2.3].

Proposition 3.

$$
(\partial / \partial r) \tilde{H}_{R}^{b}(r, t)<0
$$

and

$$
(\partial / \partial r) \tilde{K}_{R}^{b}(r, t)<0
$$

for all $t>0$ and $r \in[0, R]($ with $R<(r s p . \leqslant) \pi / \sqrt{b}$ if $b>0)$.

2. Proof of Theorem 1 and some consequences. Following [3] closely throughout, we only have to show that the transplanted heat kernels $\tilde{H}_{R}^{b}\left(r_{p}(y), t\right)$ and $\tilde{K}_{R}^{h}\left(r_{p}(y), t\right):\{p\} \times D \times\left[0, \infty\left[\rightarrow[0, \infty]\right.\right.$ satisfy $\square_{y} \tilde{H} \leqslant 0$ and $\square_{y} \tilde{K} \leqslant 0$ respectively. Here $\square_{y}=\Delta_{y}-\partial / \partial t$, where $\Delta_{y}$ is the Laplacian operating on functions on the second factor in the domain $\{p\} \times D \times[0, \infty[$.

We rewrite $\tilde{H}_{R}^{b}\left(r_{p}(y), t\right)$ as a function of $s$ and $t$, i.e. $\tilde{H}_{R}^{b}(r, t)=\tilde{H}(s(r), t)=$ $\tilde{H}(s, t)$, where

$$
s(r)= \begin{cases}1-\cos (\sqrt{b} r) & \text { if } b>0 \\ r^{2} / 2 & \text { if } b=0 \\ \cosh (\sqrt{-b} r)-1 & \text { if } b<0\end{cases}
$$

Now consider the following identity:

$$
\Delta_{y} \tilde{H}(s, t)=\tilde{H}^{\prime \prime}\left\|\operatorname{grad}_{D} s\right\|^{2}+\tilde{H}^{\prime} \Delta_{D} s,
$$

where $\tilde{H}^{\prime}=(\partial / \partial s) \tilde{H}(s, t)$.

From Proposition 2 with $F(r)=s(r)$ and $s^{\prime \prime}(r)-h_{b}(r) s^{\prime}(r) \equiv 0$ we get $\Delta_{D} s \geqslant$ $m(d s / d r) h_{b}(r)=\tilde{\Delta}_{\tilde{D}} s$, where $\tilde{\Delta}_{\tilde{D}}$ is the Laplacian on the space form $\operatorname{disc} \tilde{D}_{R}^{b}(\tilde{p})$. Proposition 3 implies $(d s / d r) \tilde{H}^{\prime} \leqslant 0$, and since $d s / d r \geqslant 0$ we get $\tilde{H}^{\prime} \Delta_{D} s \leqslant \tilde{H}^{\prime} \tilde{\Delta}_{\tilde{D}} s$. Furthermore, $\left\|\operatorname{grad}_{D} s\right\| \leqslant\left\|\operatorname{grad}_{N} s\right\|=\left\|{\operatorname{\operatorname {grad}_{\tilde {D}}}}_{\tilde{g}} s\right\|$, and finally also $\tilde{H}^{\prime \prime} \geqslant 0$ (by [3, pp. 1038-1043 and 1045-1049]). In total we therefore have from (2.2)

$$
\Delta_{y} \tilde{H}(s, t) \leqslant \tilde{H}^{\prime \prime}\left\|\widetilde{\operatorname{grad}}_{\tilde{D}} s\right\|^{2}+\tilde{H}^{\prime} \tilde{\Delta}_{\tilde{D}} s=\tilde{\Delta}_{y} \tilde{H}(s, t),
$$

so that $\square_{y} \tilde{H} \leqslant \tilde{\square}_{y} \tilde{H}=0$. 
The inequality $\square_{y} \tilde{K} \leqslant \tilde{\square}_{y} \tilde{K}=0$ for the transplanted Neumann heat kernel follows similarly from $\tilde{K}^{\prime \prime} \geqslant 0$ [3, pp. 1044 and $\left.1049-1050\right]$. The proof may now be completed by Proposition 1 of [3].

Once Theorem 1 is in hand we may now consider the series of corollaries given in [3] for similar extensions. Since the proofs of the generalized versions follow almost verbatim the space form proofs we will omit them.

COROllary A. Let $M^{m}$ be a minimally immersed submanifold of $N^{n}$. Suppose $K_{N} \leqslant b$ and let $f$ be a nonnegative subharmonic function on $M$. If $p \in M$ and $\Omega_{R}=B_{R}(p) \cap M$ for a regular ball $B_{R}(p)$, then

$$
f(p) \leqslant C^{-1}(m, b, R) \int_{\partial \Omega_{R}} f * 1,
$$

where

$$
C(m, b, R)=m \omega_{m} \cdot \begin{cases}\left(\sqrt{b}^{-1} \sin \sqrt{b} R\right)^{m-1} & \text { if } b>0, \\ R^{m-1} & \text { if } b=0, \\ \left(\sqrt{-b}^{-1} \sinh \sqrt{-b} R\right)^{m-1} & \text { if } b<0,\end{cases}
$$

and $\omega_{m}$ is the volume of the unit $m$-ball in $\mathbf{R}^{m}$.

COROllary B. Let $M^{m}$ be a minimally immersed submanifold of $N^{n}, K_{N} \leqslant b$. Let $B_{R}(p)$ be a ball in the normal range of $p \in M$. Then

$$
\operatorname{vol}\left(B_{R}(p) \cap M\right) \geqslant \operatorname{vol}\left(\tilde{D}_{\min \{R, \pi / \sqrt{b}\}}^{b}\right) .
$$

In particular, if $M$ is compact and contained in $U(p)$, then $b>0$ and

$$
\operatorname{vol}\left(M^{m}\right) \geqslant \operatorname{vol}\left(S_{b}^{m}\right)
$$

where $S_{b}^{m}$ is the round sphere of dimension $m$ and constant curvature $b$. If equality occurs in (2.6), and if $M$ is contained in $U(p)$ for every $p \in M$, then

$$
\#\left\{i \mid 0<\lambda_{i}(M) \leqslant m b\right\} \leqslant m+1,
$$

where $\left\{\lambda_{i}(m)\right\}$ is the ordered set of eigenvalues (with multiplicities) of $\Delta_{M}$.

REMARKs. The last statement follows from the generalization of Theorem 6 in [3]. The inequality (2.6) generalizes a result of B.-Y. Chen who proved it for compact minimal submanifolds of spheres (cf. [2]).

Corollary C. Let $M^{m}$ be a minimally immersed submanifold of $N$ with $K_{N} \leqslant b$. Let $D$ be a compact domain in $M$ which is contained in a regular ball $B_{R}(p)$ for some $p \in M$. Then the first Dirichlet eigenvalue $\lambda_{1}(D)$ of $\Delta_{D}$ satisfies

$$
\lambda_{1}(D) \geqslant \lambda_{1}\left(\tilde{D}_{R}^{b}\right) \geqslant m \pi^{2} / 4 R^{2} .
$$

If the first inequality is an equality, then $D$ is radial, i.e., $D$ is generated by geodesics of length $R$ from $p$. 
Furthermore, if $b \leqslant 0$, then the kth Dirichlet eigenvalue for $D$ satisfies

$$
\left(\lambda_{k}(D)\right)^{m / 2} \geqslant \frac{k \cdot(4 \pi)^{m / 2}}{e \cdot \operatorname{vol}(D)} .
$$

REMARK. The inequality $\lambda_{1}(D) \geqslant m \pi^{2} / 4 R^{2}$ was proved in [4].

\section{REFERENCES}

1. J. Cheeger and S.-T. Yau, A lower bound for the heat kernel, Comm. Pure Appl. Math. 34 (1981), $465-480$.

2. B.-Y. Chen, On the total curvature of immersed manifolds. II, Amer. J. Math. 94 (1972), 799-809.

3. S.-Y. Cheng, P. Li and S.-T. Yau, Heat equations on minimal submanifolds and their applications, Amer. J. Math. 106 (1984), 1033-1065.

4. S. Markvorsen, On the bass note of compact minimal immersions, Preprint MPI, Bonn, 1985.

Max Planck Institut fÜr Mathematik, Gottfried-Claren-Str. 26, D - 5300 Bonn 3, Federal REPUBLIC OF GERMANY

Current address: Mathematical Institute, The Technical University of Denmark, Building 303, DK-2800 Lyngby, Denmark 